\title{
Zonal flows in stellarators in an ambient radial electric field
}

\author{
Alexey Mishchenko* and Ralf Kleiber \\ Max-Planck-Institut für Plasmaphysik, \\ EURATOM-Association, D-17491 Greifswald, Germany
}

(Dated: November 12, 2012)

\begin{abstract}
The linear dynamics of zonal flows is addressed in stellarator geometry in the presence of an ambient (neoclassical) radial electric field. Global gyrokinetic particle-in-cell simulations are used to study the properties of the residual flow and its dependence on the plasma parameters and magnetic geometry. Properties of the zonal flow are compared in different magnetic geometries.
\end{abstract}

* alexey.mishchenko@ipp.mpg.de 


\section{INTRODUCTION}

Zonal flows are $\mathbf{E} \times \mathbf{B}$ flows caused by a radially varying electrostatic potential $\phi(r, t)$ driven nonlinearly by microturbulence. They play an important role in the physics of anomalous transport [1]. Zonal flows are of importance in tokamaks since the anomalous contribution to transport is dominant there. In stellarators, the neoclassical transport (caused by stellarator-specific locally-trapped particles) is significant, too. Since the early 1980s, a range of approaches to the optimization of the stellarator geometry has been developed $[2,3]$. One of the main goals of this optimization has been a reduction of the neoclassical transport. As a result, interest has also arisen to understand and, hopefully, reduce (through some optimisation procedure) the turbulent transport [4]. In tokamaks, it is known that the electric field associated with zonal flows is partially shielded due to the finite banana-orbit width of the particles (the so-called Rosenbluth-Hinton shielding [5]). It can be important to know how large the residual flow is because, to some extent, this flow indirectly indicates how effectively the turbulence can be suppressed by zonal flows. It is therefore of interest to study how the magnetic geometry affects the level of the residual zonal flow $[6,7]$.

In stellarator geometry, the Rosenbluth-Hinton shielding has been studied by Sugama and Watanabe in Ref. [8], who found that the residual zonal flow response is strongly reduced compared with the tokamak case. This reduction is associated with an additional shielding caused by the radial drift of locally-trapped particles (both ions and electrons). It has been suggested that the neoclassical optimisation may have an effect on the anomalous turbulent transport through an optimisation of the drift orbits. Indeed, it has been experimentally observed in the heliotron Large Helical Device [9] (LHD) that not only neoclassical but also anomalous transport is reduced by an inward shift of the magnetic axis. This decreases the radial drift of helically-trapped particles but also increases the unfavorable magnetic curvature which destabilizes pressure-gradient-driven instabilities such as the IonTemperature-Gradient driven (ITG) modes (see Ref. [10] and the papers referenced therein). It was argued that the larger linear growth rates of ITG modes in the LHD configuration with an inward shift of the magnetic axis can be compensated by more effective turbulence suppression through larger zonal flows. This effect of the drift optimisation in LHD on microturbulence was studied numerically with a flux-tube Eulerian code GKV [8, 11].

In Refs. $[12,13]$, the initial zonal-flow problem was solved using Laplace transformation, 
and it was found that the linear zonal flow response is oscillatory in stellarator geometry (in contrast to tokamaks). These oscillations are caused by the bounce-averaged radial drift motion of the locally-trapped particles [12]. They are damped by a Landau damping mechanism since the radial drift depends on the particle energy, which varies over the trapped-particle population, thus producing phase mixing [13]. In addition to the damped oscillations, the linear zonal-flow response contains an algebraically-damped component and a residual flow (studied in Refs. [8] and [13]). The zonal flow oscillations have been observed numerically both in global particle-in-cell (PIC) simulations using the EUTERPE code [13, 14] and in flux-tube Eulerian simulations using the GENE code $[13,15]$. They appear to be more pronounced in optimised configuration such as the Wendelstein 7-X (W7-X) [16] whereas their damping is rather strong in a more "classical" heliotron (such as the LHD device).

In Ref. [17], the effect of the neoclassical electric field (needed in order to satisfy the ambipolarity condition in non-symmetric plasmas) on the residual zonal flow was considered. It was found that, in contrast to tokamaks, the residual level is strongly affected (increased) by the ambient electric field. A similar conclusion was drawn in Ref. [18] using an actionangle formalism. A more favourable ion-mass dependence than the conventional gyro-Bohm scaling of the zonal-flow response has been found in Ref. [17], and collisionless detrapping (producing so-called transitioning particles) was included in the theory. Numerically, the role of the ambient electric field was studied with the poloidally-global (but local in the radial direction) Eulerian code GKV in the LHD magnetic field [17, 19, 20]. The fullyglobal EUTERPE code has been implemented [14] in the magnetic fields of W7-X, LHD and Helically Symmetric Experiment (HSX).

In the present paper, we extend the analytical approach of Ref. [17] to include the transient dynamics (the zonal flow oscillations). For the numerical simulations, we employ the fully-global (both in the poloidal and radial directions) gyrokinetic particle-in-cell code EUTERPE [14, 21]. As has been observed in Refs. [13, 15], zonal flow properties can strongly depend on the magnetic configuration when the ambient electric field is assumed to vanish. Here, we study (numerically) which effect the magnetic geometry has on the zonal flow if the ambient (neoclassical) radial electric field is finite. Using EUTERPE, we compare linear zonal flow properties in two distinct stellarator configurations: LHD, which can be considered as a close relative to the classical stellarator configuration, and the drift-optimized stellarator Wendelstein 7-X [16] (W7-X). By comparing these configurations, the effect of 
the ambient electric field on the zonal flow properties (such as the asymptotic residual level) and on the transient dynamics (e. g. the zonal-flow oscillation frequency and the damping rate) are considered. The role of the neoclassical electric field profile (shear etc) is also addressed.

The structure of the paper is as follows. In Sec. II, basic equations are presented and the zonal flow response is derived. In Sec. III, the gyrokinetic code EUTERPE is employed to perform simulations in the stellarator configurations mentioned above. The simulation results are discussed and conclusions are drawn in Sec. IV.

\section{THEORY}

\section{A. Zonal-flow gyrokinetic equation in Boozer coordinates}

An elaborate theory of the residual zonal flows in helical systems with radial electric fields has been developed by Sugama and Watanabe in Ref. [17] using a multiple-helicity model of the magnetic field. An alternative approach based on the action-angle formalism was suggested by Mynick and Boozer [18]. Here, we derive the linear zonal flow response for general non-axisymmetric magnetic field using Boozer coordinates. In addition to the residual flow properties studied in Refs. $[17,18]$, we also consider transient dynamics (the zonal flow oscillations and damping).

The magnetic field $\mathbf{B}$ in Boozer coordinates can be written as follows [22, 23]:

$$
\mathbf{B}=\nabla \psi \times \nabla \alpha=I \nabla \zeta+\widetilde{\beta} \nabla \psi
$$

Here, $\psi=\psi_{0} s$ is the toroidal flux with $\psi_{0}$ being the toroidal flux at the plasma edge and $s$ the flux-surface label, $\alpha$ is a field-line label, $\zeta=\int B / I \mathrm{~d} l$ is the coordinate along the field line (integration is performed along this line), and $I=I(\psi)$. We consider a stellarator plasma with the equilibrium magnetic field $\mathbf{B}$, an ambient electrostatic potential $\Phi(s)$ (e.g. of neoclassical origin) and a self-consistent zonal-flow potential $\phi(s, t)$ (we neglect the sidebands). Any effects of the plasma pressure profile are neglected (the pressure is taken to be flat) as are electromagnetic effects. Then, the linearized gyrokinetic equation can be written as follows:

$$
\frac{\partial f_{1}}{\partial t}+v_{\|} \nabla_{\|} f_{1}+\mathbf{v}_{d} \cdot \nabla f_{1}+\mathbf{u}_{E} \cdot \nabla f_{1}=-f_{0} \mathbf{v}_{d} \cdot \nabla\langle\hat{\phi}\rangle, \quad \hat{\phi}=\frac{e \phi}{T}
$$


Here, $f_{1}$ is the perturbed distribution function, $f_{0}$ is assumed to be Maxwellian, $\langle\hat{\phi}\rangle$ is the gyro-averaged normalized perturbed potential, $v_{\|}=\mathbf{v} \cdot \mathbf{B} / B$ is the parallel particle velocity, $\mathbf{v}_{d}=\rho_{\|} \nabla \times\left(v_{\|} \mathbf{B} / B\right)$ is the magnetic drift velocity, $\rho_{\|}=m v_{\|} /(e B)$ is the "parallel gyroradius" and $\mathbf{u}_{E}=\mathbf{B} \times \nabla \Phi / B^{2}$ is the $\mathbf{E} \times \mathbf{B}$ velocity associated with the background electric field. The unperturbed particle energy $\epsilon=m v^{2} / 2+e \Phi$ is used as an independent variable. The gyrokinetic equation is to be solved with the initial condition $f_{1}(t=0) \neq 0$ and $\phi(t=0)=0$. Physically, it means that we study the response of the plasma to an initial density perturbation. Substituting Eq. (1), the kinetic equation can be written in the form (see Ref. [23]):

$$
\frac{\partial h}{\partial t}+\frac{v_{\|} B}{I} \frac{\partial h}{\partial \zeta}+\left(\Omega_{E}-\frac{v_{\|} B}{\psi_{0}} \frac{\partial \rho_{\|}}{\partial s}\right) \frac{\partial h}{\partial \alpha}+\frac{v_{\|} B}{\psi_{0}} \frac{\partial \rho_{\|}}{\partial \alpha} \frac{\partial h}{\partial s}=\frac{\partial\langle\hat{\phi}\rangle}{\partial t}
$$

where $h=f_{1}+f_{0}\langle\hat{\phi}\rangle$ and $\Omega_{E}=\Phi^{\prime} / \psi_{0}$.

\section{B. Effect of the shearless ambient electric field on zonal-flow dynamics}

We assume that the zonal-flow radial scale is much smaller than the plasma minor radius and neglect the shear of the ambient radial electric field (shearless profile). In this case, an eikonal approximation can be employed in the radial direction [i. e. we assume $h=$ $h_{k} \exp \left(i \int k_{r} \mathrm{~d} r\right)$ and $\left.\hat{\phi}=\phi_{k} \exp \left(i \int k_{r} \mathrm{~d} r\right)\right]$. Then, one can write the kinetic equation to the lowest order in $1 /\left(k_{r} r_{a}\right)$ (here $r_{a}$ is the minor radius) as follows:

$$
\frac{\partial h_{k}}{\partial t}+\frac{v_{\|} B}{I} \frac{\partial h_{k}}{\partial \zeta}+\left(\Omega_{E}-\frac{v_{\|} B}{\psi_{0}} \frac{\partial \rho_{\|}}{\partial s}\right) \frac{\partial h_{k}}{\partial \alpha}+i \omega_{r} h_{k}=\frac{\partial \phi_{k}}{\partial t} J_{0 g}
$$

Here, $J_{0 g}=J_{0}\left(k_{r} \rho_{s}\right)$ is the gyro-average in the eikonal representation (with $\rho_{s}$ the particle gyroradius), $\omega_{r}=k_{r} v_{r}$ with $v_{r}=\left(v_{\|} B / \psi_{0}\right) \partial \rho_{\|} / \partial \alpha$ the radial drift velocity and $k_{r}$ the radial wave number. The bounce-average operation in Boozer coordinates can be defined as follows:

$$
\begin{aligned}
& \langle Q\rangle_{b}=\hat{\omega}_{b} \oint Q \frac{I}{v_{\|} B} \mathrm{~d} \zeta, \quad \hat{\omega}_{b}=\left(\oint \frac{I}{v_{\|} B} \mathrm{~d} \zeta\right)^{-1} \\
& I \psi_{0}=B^{2} \sqrt{g}, \quad \frac{1}{\sqrt{g}}=\left(\nabla s_{V} \times \nabla \alpha\right) \cdot \nabla \zeta
\end{aligned}
$$

Here, as usual, the integration is performed between the bounce points for the reflected particles with well-defined bounce motion and over the entire flux surface for the passing 
particles. The radial drift velocity can be split into a bounce-dependent part $\widetilde{v}_{r}=v_{\|} \nabla_{\|} \delta_{r}$ and a bounce-averaged part $\left\langle v_{r}\right\rangle_{b}=m \hat{\omega}_{b} /\left(e \psi_{0}\right) \partial J / \partial \alpha$ (which is non-zero for most locallytrapped particles). Here, $\delta_{r}=\int_{l} \mathrm{~d} l / v_{\|}\left(v_{r}-\left\langle v_{r}\right\rangle_{b}\right)$ represents the radial displacement of the gyrocenter from the bounce-averaged radial position, $J=\oint v_{\|} \mathrm{d} l$ is the second adiabatic invariant and $\mathrm{d} l=(I / B) \mathrm{d} \zeta$ is the length element along the magnetic field line. In this notation, the gyrokinetic equation takes the form:

$$
\frac{\partial \hat{h}_{k}}{\partial t}+\frac{v_{\|} B}{I} \frac{\partial \hat{h}_{k}}{\partial \zeta}+\left(\Omega_{E}+\omega_{\alpha}\right) \frac{\partial \hat{h}_{k}}{\partial \alpha}+i\left\langle\omega_{r}\right\rangle_{b} \hat{h}_{k}=\frac{\partial \phi_{k}}{\partial t} J_{0 g} \exp \left(i k_{r} \delta_{r}\right)
$$

Here $\hat{h}_{k}=h_{k} \exp \left(i k_{r} \delta_{r}\right)$ and $\omega_{\alpha}=-\left(v_{\|} B / \psi_{0}\right) \partial \rho_{\|} / \partial s$, we have neglected $\partial \delta_{r} / \partial \alpha$ for simplicity (although this neglect cannot rigorously be justified in general stellarator geometry). In this section, we will also assume that the ambient electric field is strong enough so that $\omega_{\alpha}$ can be neglected, too (compared with $\left.\Omega_{E}\right)$. The gyrokinetic equation coupled to the quasineutrality equation describes the linear multiple time-scale dynamics of the radial self-consistent electric field in stellarator geometry. The relevant time scales include the bounce time $\hat{\omega}_{b} t \sim 1$, the radial drift time $\left\langle\omega_{r}\right\rangle_{b} t \sim 1$ and the poloidal $\mathbf{E} \times \mathbf{B}$-drift time $\Omega_{E} t \sim 1$ (which can be larger or smaller than the magnetic drift time depending on the Mach number $M=u_{E} / v_{\text {th } i}$ with $u_{E}$ the $\mathbf{E} \times \mathbf{B}$ velocity and $v_{\text {th } i}$ the thermal speed of ions). On the fastest bounce time scale (since the gyro-time scale is excluded from the gyrokinetic equation), the Geodesic Acoustic Mode (GAM) dynamics occurs (corresponding to $\hat{\omega}_{b} t \sim 1$ but $\left.\left\langle\omega_{r}\right\rangle_{b} t \ll 1\right)$. This dynamics involves non-zonal components of the self-consistent potential which are not considered here. As has been shown in Ref. [8], the GAM dynamics in stellarators is qualitatively similar to that in tokamaks (the stellarator-specific drifts do not alter the physics qualitatively on these fast time scales). Assuming the time scales of interest to be much longer than the bounce time (this filters out the GAM oscillations [8]), one can write the gyrokinetic equation to lowest order in $1 /\left(\hat{\omega}_{b} t\right)$ as follows [here we employ the bounce-average in Eq. (7) and define $\left.J_{0 b}=\left\langle\exp \left(i k_{r} \delta_{r}\right)\right\rangle_{b}\right]$ :

$$
\frac{\partial \hat{h}_{k}^{(0)}}{\partial t}+\Omega_{E} \frac{\partial \hat{h}_{k}^{(0)}}{\partial \alpha}+i\left\langle\omega_{r}\right\rangle_{b} \hat{h}_{k}^{(0)}=\frac{\partial \phi_{k}}{\partial t} J_{0 g} J_{0 b}
$$

This equation (coupled to the quasineutrality equation) describes the slow "residual" dynamics of the perturbed radial electric field. One way to solve Eq. (8) is to introduce the following coordinate transformation:

$$
\eta=\alpha-\Omega_{E} t, \quad \tau=t
$$


In these coordinates, the kinetic equation takes the form:

$$
\frac{\partial \hat{h}_{k}^{(0)}}{\partial \tau}+i\left\langle\omega_{r}\right\rangle_{b}(\eta, \tau) \hat{h}_{k}^{(0)}=\frac{\partial \hat{\phi}}{\partial \tau} J_{0 g} J_{0 b}
$$

The solution of this equation is straightforward:

$$
\hat{h}_{k}^{(0)}=\hat{h}_{k}^{(0)}(\tau=0)+\int_{0}^{\tau} \mathrm{d} \tau^{\prime} \frac{\partial \phi_{k}}{\partial \tau^{\prime}} J_{0 g} J_{0 b} \exp \left\{i k_{r}\left[\Delta_{r}\left(\tau^{\prime}\right)-\Delta_{r}(\tau)\right]\right\}
$$

Here, $\Delta_{r}(\tau)=\int_{0}^{\tau}\left\langle v_{r}\right\rangle_{b}(\tau) \mathrm{d} \tau^{\prime}$ is a radial width of the particle drift orbit. Obviously, in the absence of the ambient radial electric field $\left\langle v_{r}\right\rangle_{b}=\operatorname{const}(\tau)$ so that $\Delta_{r}=\left\langle v_{r}\right\rangle_{b} \tau$. This results in a qualitatively different dynamics of the self-consistent electrostatic potential $[8,13]$.

The solution of the kinetic equation has to be substituted into the quasineutrality condition. Assuming the electron response to be purely adiabatic and using the definition of the non-adiabatic part of the perturbed ion distribution function $h=f_{1}+f_{0}\langle\hat{\phi}\rangle$, we obtain the quasinetrality equation in our notation as follows:

$$
-n_{0} \phi_{k}+\left\langle\int \mathrm{d}^{3} v h_{k} f_{0} J_{0 g}\right\rangle^{(\text {ions })}=0
$$

Here, the flux-surface average is defined as usual [the Jacobian $\sqrt{g}$ has been defined in Eq. (6)]:

$$
\langle Q\rangle=\frac{1}{V^{\prime}} \int Q \sqrt{g} \mathrm{~d} \zeta \mathrm{d} \alpha, \quad V^{\prime}=\int \sqrt{g} \mathrm{~d} \zeta \mathrm{d} \alpha
$$

The resulting equation determines the evolution of the zonal flow potential. It has the form:

$$
-n_{0} \phi_{k}+\left\langle k_{r}^{2} \rho_{i}^{2}\right\rangle n_{0} \phi_{0}+\int_{0}^{\tau} \mathrm{d} \tau^{\prime} \frac{\partial \phi_{k}}{\partial \tau^{\prime}}\left\{J_{0 g}^{2} J_{0 b} \bar{J}_{0 b} \exp \left(i k_{r}\left[\Delta_{r}\left(\tau^{\prime}\right)-\Delta_{r}(\tau)\right]\right)\right\}=0,
$$

Here, the phase-space average is $\{Q\}=\left\langle\int \mathrm{d}^{3} v f_{0} Q\right\rangle$ and $\bar{J}_{0 b}=\left\langle-\exp \left(i k_{r} \delta_{r}\right)\right\rangle_{b}$. To include the effect of the transitioning particles, one can modify this definition introducing probabilities for a particle to be either trapped or passing (as it has been done in Ref. [17]). Here, we skip this effect for simplicity. Also, we have employed the long-wavelength approximation in the polarization density $\left(k_{r} \rho_{i} \ll 1\right)$ and, as has already been mentioned, assumed the electron response to be purely adiabatic. Clearly, in stellarator geometry there is also a non-adiabatic electron contribution to the zonal-flow response (in contrast to the tokamak case) because the width of the electron drift orbit $\Delta_{r}$ is comparable to the ion one $[8,11,13]$. However, inclusion of the non-adiabatic electron part does not make any qualitative difference. In addition, the 
gyrokinetic simulations of the zonal flow response usually employ the adiabatic-electron approximation (also in this paper). The initial condition for the perturbed ion distribution function is chosen, as usual [8], so that the initial electrostatic field perturbation is shielded by the classical polarization: $f_{1 i}(\tau=0)=\left\langle k_{r}^{2} \rho_{i}^{2}\right\rangle f_{0} \phi_{0}$.

To solve Eq. (14), we assume that the bounce-averaged drift velocity is given by the simple expression $\left\langle v_{r}\right\rangle_{b}=V_{r}(s, \mu, v) \sin \alpha$ and neglect all other $\alpha$-dependencies (here, $\mu$ denotes the magnetic moment). Note that this approximation of the bounce-averaged radial drift velocity is consistent with the magnetic field model used in the so-called $\sigma$-optimization (see e. g. Ref. [24] for details). Then, the field-evolution equation takes the form:

$$
-n_{0} \phi_{k}+\left\langle k_{r}^{2} \rho_{i}^{2}\right\rangle n_{0} \phi_{0}+\int_{0}^{\tau} \mathrm{d} \tau^{\prime} \frac{\partial \phi_{k}}{\partial \tau^{\prime}}\left\{J_{0 g}^{2} J_{0 b} \bar{J}_{0 b} J_{0}\left[K\left(\tau-\tau^{\prime}\right) d_{r}\right]\right\}=0,
$$

where $J_{0}\left[K(\tau) d_{r}\right]$ is a Bessel function and

$$
K(\tau)=2 k_{r} \sin \left(\frac{\Omega_{E} \tau}{2}\right), \quad d_{r}=V_{r} / \Omega_{E}
$$

Note that the "averaging factors" (the Bessel functions) similar to Eq. (15) have appeared also in Ref. [18] derived by Mynick and Boozer using the action-angle formalism.

In the long-wavelength approximation (assuming $k_{r} \rho_{i}, k_{r} \delta_{r}, k_{r} d_{r} \ll 1$ ), the equation for the electrostatic potential has the form (here $\left\{\hat{\delta}_{r}^{2}\right\}=\left\{\left\langle\delta_{r}^{2}\right\rangle_{b}-\left\langle\delta_{r}\right\rangle_{b}^{2}\right\}$ and $\left\{\hat{d}_{r}^{2}\right\}=\left\{d_{r}^{2} / 2\right\}$ ):

$$
\left(1+\frac{\left\{\hat{\delta}_{r}^{2}\right\}}{\left\langle\rho_{i}^{2}\right\rangle}\right) \phi_{k}(t)+\frac{\left\{\hat{d}_{r}^{2}\right\}}{\left\langle\rho_{i}^{2}\right\rangle} \int_{0}^{t} \phi_{k}(t-\tau) \sin \left(\Omega_{E} \tau\right) \Omega_{E} \mathrm{~d} \tau=\phi_{0}
$$

This equation is straightforward to solve using the Laplace transform. The solution is

$$
\frac{\phi_{k}(t)}{\phi_{0}}=\frac{1}{\hat{R}}\left[1+\frac{\hat{d}^{2}}{\hat{R}-\hat{d}^{2}} \cos \left(\omega_{Z F} t\right)\right]
$$

with

$$
\hat{R}=1+\frac{\left\{\hat{\delta}_{r}^{2}\right\}}{\left\langle\rho_{i}^{2}\right\rangle}+\frac{\left\{\hat{d}_{r}^{2}\right\}}{\left\langle\rho_{i}^{2}\right\rangle}, \quad \hat{d}^{2}=\frac{\left\{\hat{d}_{r}^{2}\right\}}{\left\langle\rho_{i}^{2}\right\rangle}, \quad \omega_{Z F}=\Omega_{E} \sqrt{\frac{\hat{R}}{\hat{R}-\hat{d}^{2}}}
$$

The resulting zonal flow represents oscillations around the residual level $1 / \hat{R}$. This residual level coincides with that derived by Sugama and Watanabe [17] when the transitioning orbits are neglected (see the discussion below). The constant part of the residual flow increases with the electric field (see [17] for a detailed discussion on the scalings), and so does the frequency of the oscillations $\omega_{Z F}$ (it is proportional to $\Omega_{E}$ ), but the oscillation amplitude 
decreases with the Mach number $M=u_{E} / v_{\text {thi } i}$ [through the dependence of $\hat{d}$ on $\Omega_{E}$ in Eq. (18)]. It is interesting that the oscillations considered here show no damping. This happens since we have assumed that the dominant part of the poloidal drift is due to the $\mathbf{E} \times \mathbf{B}$-motion which is independent of the particle velocity (hence, no phase mixing occurs in velocity space). The situation would change if we included the poloidal magnetic drift into the consideration. Note however, that Eq. (18) indicates that a phase mixing in real space also possible if the ambient radial electric field and, hence, the zonal flow frequency have a radial dependence $\omega_{\mathrm{ZF}}=\omega_{\mathrm{ZF}}(s)$.

In Ref. [17], Sugama and Watanabe derived the expression for the residual zonal flow, too. However, they solved the bounce-averaged kinetic equation (8) asymptotically assuming $\Omega_{E} t \gg 1$. Their solution contains the zonal flow residual part but neglects (unsurprisingly) the zonal-flow oscillations with the frequency close to $\Omega_{E}$. The zonal flow residual derived by Sugama and Watanabe (see, for example, Eq. (33) in Ref. [17]) can be shown to coincide with our result when the effects of the transitioning particles (collisionless detrapping) are neglected (as we did in our derivation). Technically, it means a simple definition for the poloidal average instead of the elaborated one (expressed in terms of the transition probabilities) which has been employed in [17] (for a simple poloidal average, one can write $\left.\left\langle\Delta_{r}\right\rangle_{p o} \sim \int_{0}^{2 \pi} V_{r}(\alpha) \mathrm{d} \alpha=0\right)$. Our expression for the residual part of the zonal flow [see Eq. (19)] agrees also with that of Mynick and Boozer (see e. g. Eq. (16b) of Ref. [18]). The classical Rosenbluth-Hinton expression for the residual zonal flow in the tokamak limit follows immediately from Eq. (19), too $\left(\left\{\hat{d}_{r}^{2}\right\}=0\right.$ in this case).

\section{Damping of zonal flow oscillations}

Consider now the situation where the magnetic contribution to the poloidal drift of the reflected (helically-trapped) particles is comparable to the $\mathbf{E} \times \mathbf{B}$ contribution. In contrast to $\Omega_{E}$, the magnetic poloidal drift is related to $\omega_{\alpha}(v)$, which depends on the particle velocity and, physically, it is natural to expect some kind of phase mixing (damping of the oscillations) resulting from this dependency. Formally, we cannot neglect $\omega_{\alpha}$ compared with $\Omega_{E}$ in Eq. (7). Hence, Eq. (8) must be replaced by

$$
\frac{\partial \hat{h}_{k}^{(0)}}{\partial t}+\left(\Omega_{E}+\left\langle\omega_{\alpha}\right\rangle_{b}\right) \frac{\partial \hat{h}_{k}^{(0)}}{\partial \alpha}+i\left\langle\omega_{r}\right\rangle_{b} \hat{h}_{k}^{(0)}=\frac{\partial \phi_{k}}{\partial t} J_{0 g} J_{0 b}
$$


Proceeding with the solution in the same way as in Sec. II B, we obtain the Laplace transform of the electrostatic potential:

$$
\Phi(p)=\frac{\phi_{0}}{p}\left[1+\frac{\left\{\hat{\delta}_{r}^{2}\right\}}{\left\langle\rho_{i}^{2}\right\rangle}+I(p)\right]^{-1}
$$

with

$$
I(p)=\frac{2 F_{T}^{\prime} v_{t h}}{V^{\prime} \sqrt{\pi}} \int \mathrm{d} \theta \int \mathrm{d} \lambda \hat{\tau}_{b} \frac{\bar{d}_{r}^{2}}{\left\langle\rho_{i}^{2}\right\rangle} \int_{0}^{\infty} \frac{x^{6}\left(\Omega_{E}+\bar{\omega}_{\alpha} x^{2}\right)^{2} e^{-x^{2}} \mathrm{~d} x}{p^{2}+\left(\Omega_{E}+\bar{\omega}_{\alpha} x^{2}\right)^{2}}
$$

Note that the poloidal magnetic drift frequency is expressed as $\left\langle\omega_{\alpha}\right\rangle_{b}=\bar{\omega}_{\alpha} x^{2}$ where $x=v / v_{t h}$ and $\bar{\omega}_{\alpha}>0$ is assumed (here, we neglect the $\alpha$-dependent part of $\left\langle\omega_{\alpha}\right\rangle_{b}$ ). For convinience, we now define a function $G(p)$ as follows:

$$
G(p)=\int_{0}^{\infty} \mathcal{N}(p, x) \mathrm{d} x, \quad \mathcal{N}(p, x)=\frac{x^{6}\left(\Omega_{E}+\bar{\omega}_{\alpha} x^{2}\right)^{2} e^{-x^{2}}}{p^{2}+\left(\Omega_{E}+\bar{\omega}_{\alpha} x^{2}\right)^{2}}
$$

In this notation, Eq. (22) takes the form:

$$
I(p)=\frac{2 F_{T}^{\prime} v_{t h}}{V^{\prime} \sqrt{\pi}} \int \mathrm{d} \theta \int \mathrm{d} \lambda \hat{\tau}_{b} \frac{\bar{d}_{r}^{2}}{\left\langle\rho_{i}^{2}\right\rangle} G(p)
$$

Note that in the complex $x$-plane, the function $\mathcal{N}(p, x)$ defined in Eq. (23) has four poles:

$$
x_{1,2}= \pm i\left(\frac{\Omega_{E}+i p}{\bar{\omega}_{\alpha}}\right)^{1 / 2} ; \quad x_{3,4}= \pm i\left(\frac{\Omega_{E}-i p}{\bar{\omega}_{\alpha}}\right)^{1 / 2}
$$

The inverse Laplace transform (here $a>0$ is a positive real number)

$$
\phi(t)=\frac{1}{2 \pi i} \int_{-i \infty+a}^{i \infty+a} \Phi(p) e^{p t} \mathrm{~d} p
$$

is to be taken along the contour shown in Fig. 1. Since this integral can hardly be evaluated analytcally, we deform the contour as shown in Fig. 2 (this manipulation is in the spirit of Landau damping derivation and has already been used for zonal flows in Ref. [13]). Singularities of $\Phi(p)$ in the complex plane include the simple pole in $p=0$ (defining the residual flow) and the singularities related to the poles of the function $\mathcal{N}(p, x)$ defined in Eq. (23). In contrast to the usual Landau damping problem, the poles Eq. (25) are proportional to $\left(p \pm i \Omega_{E}\right)^{1 / 2}$ instead of $p$. Hence, the poles of $\mathcal{N}(p, x)$ in the complex $x$-plane generate branch points at $p= \pm i \Omega_{E}$ as singularities of $\Phi(p)$ in the complex $p$-plane when shifting the inverse Laplace transform integration contour to the left as shown in Fig. 2.

Mathematically, the presence of these branch points causes damped/oscillatory behavior of linear zonal flows in non-axisymmetric geometry. In order to see why, we note that an 
analytic continutation for the functions $G(p)$ and, consequently, $I(p)$ is needed when the Laplace integration contour (Fig. 1) is deformed. This analytic continuation is non-trivial only when a pole of $\mathcal{N}(p, x)$ [see Eq. (25)] crosses the positive half of the real axis in the complex $x$-plane when the integration contour in the complex $p$-plane is shifted to the left (as shown in Fig. 2). One can show that for the upper cut, such a crossing happens only on the upper branch. For the lower cut, only the lower branch is involved (the corresponding integration contours both in the complex $p$-plane and the complex $x$-plane are shown in Figs. 3, 4 and 5).

Thus, for the upper branch of the upper cut one can write:

$$
G(p)=\hat{G}(p)+\frac{\pi}{4} \hat{p}_{u}^{5 / 2} \exp \left(i \hat{p}_{u}+i \pi / 4\right)\left(\frac{\Omega_{E}}{\bar{\omega}_{\alpha}}-i \hat{p}_{u}\right), \quad \hat{p}_{u}=\frac{p-i \Omega_{E}}{\bar{\omega}_{\alpha}}, \quad \arg \left(\hat{p}_{u}\right)=\pi
$$

Here, the quantitiy $\hat{G}(p)$ is defined below in Eq. (29). The pole in the velocity integrals that is to be taken into account during the analytical continuation is $x_{2}=\hat{p}_{u}^{1 / 2} \exp (-i \pi / 4)$. On the lower branch of the lower cut, one obtains:

$$
G(p)=\hat{G}(p)+\frac{\pi}{4} \hat{p}_{l}^{5 / 2} \exp \left(-i \hat{p}_{l}-i \pi / 4\right)\left(\frac{\Omega_{E}}{\bar{\omega}_{\alpha}}-i \hat{p}_{l}\right), \quad \hat{p}_{l}=\frac{p+i \Omega_{E}}{\bar{\omega}_{\alpha}}, \quad \arg \left(\hat{p}_{l}\right)=-\pi
$$

Here, the corresponding pole on the complex velocity plane is $x_{3}=\hat{p}_{l}^{1 / 2} \exp (i \pi / 4)$. Otherwise (for the lower branch of the upper cut and the upper branch of the lower cut), the analytic continuation is trivial:

$$
G(p)=\hat{G}(p)=P . V . \int_{0}^{\infty} \frac{x^{6}\left(\Omega_{E}+\bar{\omega}_{\alpha} x^{2}\right)^{2} e^{-x^{2}} \mathrm{~d} x}{p^{2}+\left(\Omega_{E}+\bar{\omega}_{\alpha} x^{2}\right)^{2}}
$$

Substituting the analytic continuation of $G(p)$ into Eq. (23) and performing the inverse Laplace transform along the deformed integration contour (shown in Fig. 2), one obtains for large times $\Omega_{E} t>1$ :

$$
\frac{\phi(t)}{\phi(t=0)}=\left(1+\frac{\left\{\hat{\delta}_{r}^{2}\right\}}{\left\langle\rho_{i}^{2}\right\rangle}+\frac{\left\{\hat{d}_{r}^{2}\right\}}{\left\langle\rho_{i}^{2}\right\rangle}\right)^{-1}\left[1+\frac{c_{1}}{t^{7 / 2}} \sin \left(\Omega_{E} t+\pi / 4\right)\right]
$$

Here, the Watson Lemma has been used to compute the integral along the branch cut (small values of $\hat{p}_{u}$ and $\hat{p}_{l}$ give the dominant contribution).

One sees that the zonal flow evolution includes a constant residual and an oscillatory algebraically-damped parts. The oscillation frequency coincides with the frequency of the 
$\mathbf{E} \times \mathbf{B}$ rotation. The quantity $c_{1}$ depends on the magnetic configuration and on the ambient magnetic field:

$$
\begin{aligned}
& c_{1}=\left[1+\frac{\left\{\hat{\delta}_{r}^{2}\right\}}{\left\langle\rho_{i}^{2}\right\rangle}+\hat{I}_{0}\right]^{-2}\left(\frac{\left\{\hat{\delta}_{r}^{2}\right\}}{\left\langle\rho_{i}^{2}\right\rangle}+\hat{I}_{0}\right) \frac{F_{T}^{\prime} v_{t h}}{2 V^{\prime} \sqrt{\pi}} \int \mathrm{d} \theta \int \mathrm{d} \lambda \hat{\tau}_{b} \frac{\bar{d}_{r}^{2}}{\left\langle\rho_{i}^{2}\right\rangle} \frac{\Gamma(7 / 2)}{\bar{\omega}_{\alpha}^{7 / 2}} \\
& \hat{I}_{0}=\frac{2 F_{T}^{\prime} v_{t h}}{V^{\prime} \sqrt{\pi}} \int \mathrm{d} \theta \int \mathrm{d} \lambda \hat{\tau}_{b} \frac{\bar{d}_{r}^{2}}{\left\langle\rho_{i}^{2}\right\rangle} \int_{0}^{\infty} \frac{x^{6}\left(\Omega_{E}+\bar{\omega}_{\alpha} x^{2}\right)^{2} e^{-x^{2} \mathrm{~d} x}}{\bar{\omega}_{\alpha}\left(2 \Omega_{E}+\bar{\omega}_{\alpha} x^{2}\right)}
\end{aligned}
$$

Depending on the quantity $c_{1}$ (or, more precisely, on the ratio between $\Omega_{E}$ and $\bar{\omega}_{\alpha}$ ), the ZF oscillations may seem almost undamped (this is the case for vanishingly small $\bar{\omega}_{\alpha}$ considered in the previous section) or heavily damped, since the power of the algebraic damping is rather large $\left(\phi \sim t^{-7 / 2}\right)$. One can speculate that different magnetic configurations may show rather disparate zonal flow responses, depending on the magnetic field optimization (which usually reduces the magnetic drift velocity).

\section{SIMULATIONS}

In this section, global gyrokinetic simulations using a global particle-in-cell code EUTERPE $[14,21]$ will be presented. The purpose of these simulations will be to confirm (at least qualitatively) and extend our analytical findings from the previous Section. Before proceeding with numerics, let us summarize our analytical findings:

1. The linear zonal flow response consists of a constant (residual) and an oscillatory parts.

2. The residual part increases with the ambient radial electric field (this is consistent with Ref. [17]).

3. The residual level does not depend on the perpendicular wave number if the ambient electric field is finite (also discussed in Ref. [17]).

4. The frequency of the oscillations is close to the frequency of the poloidal $\mathbf{E} \times \mathbf{B}$ drift motion.

5. The amplitude of the oscillations decreases with the ambient electric field.

6. The oscillations are essentially undamped when the magnetic contribution to the poloidal drift is negligible compared with $\Omega_{E}$ (no phase mixing in velocity space). 
This case occurs in W7-X (details will be discussed in this Section). The oscillations are heavily damped when the magnetic poloidal drift frequency is comparable to the $\mathbf{E} \times \mathbf{B}$ contribution (strong phase mixing in velocity space). This situation appears to be typical for the LHD configuration with the major radius $R_{0}=3.75 \mathrm{~m}$ (see below).

7. A phase mixing in the real space is also possible (although not yet explicitely considered) since the oscillation frequency can be a strong function of the radial coordinate (if the shear of the ambient electric field is large enough).

Now, consider the standard W7-X geometry [16]. The plasma temperature and density profiles are assumed to be flat, the electrons are adiabatic, only the electrostatic part $\phi$ of the perturbation is considered. As an initial condition for the ion perturbed distribution function, we employ $\delta f_{i}(t=0) \sim \cos \left(\pi \rho^{2} / 2\right)$ with $\rho=\sqrt{\psi / \psi_{0}}$ (recall that $\psi$ is the toroidal flux). We are interested in the self-consistent response of the gyrokinetic non-axisymmetric plasma in the presence of a constant ambient radial electric field corresponding to $\Omega_{E} / \omega_{\mathrm{c} i}=$ $3.996 \times 10^{-5}$ (here $\omega_{c i}$ is the ion cyclotron frequency). The resulting radial profile of the electrostatic potential (corresponding to the end of the simulation) is shown in Fig. 6. One can see that it resembles, essentially, the initial cosine profile. The time evolution of $\phi^{\prime}=$ $\partial \phi / \partial s$ at different radial positions is shown in Fig. 7. One can see some GAMs at the very beginning (note that GAMs are very weak in W7-X, see also Refs. [13, 14]). At later times, a low-frequency almost undamped mode is observed. The frequency of this mode is close to $\Omega_{E}$, in agreement with the analytical indications mentioned above. It is striking how the plasma oscillates with the same frequency at all radial locations. Of course, this is related to the flat profile of the ambient electric field chosen in this simulation.

It is interesting to study how the zonal flow evolution changes when the ambient electric field increases. This is shown in Fig. 8, where the evolution of $\phi^{\prime}$ at the same radial location is plotted for different Mach numbers $M=u_{E} / v_{\text {th } i}$. Here, one can see that the residual level, the oscillation frequency and the oscillation amplitude - all change (as expected). The change in the residual is shown in Fig. 9 (this result is consistent with Ref. [17]). The dependence of the frequency is plotted in Fig. 10. Again, it is striking how robustly the simulations reproduce $\omega_{Z F} \approx \Omega_{E}$ for the magnetic configuration considered.

Now, let us consider how the zonal flow depends on the radial wave number. Strictly speaking, there is no explicit radial wave number in global simulations. We can, however, 
initialize the perturbed ion distribution functions with various characteristic radial scales. Thus, we choose $\delta f(t=0) \sim \cos \left(k \psi / \psi_{0}\right)$ and compare the $k$-dependence for cases with small Mach number (corresponding to $\Omega_{E} / \omega_{c i}=0.8 \times 10^{-5}$ or, equivalently, $u_{E} / v_{\text {th } i}=0.0036$ ) and "moderate" Mach number (here $\Omega_{E} / \omega_{c i}=4 \times 10^{-5}$ and $u_{E} / v_{\text {th } i}=0.018$ ). The evolution in the moderate-Mach-number case is shown in Fig. 11 and the global radial profiles of the perturbed electrostatic potential in Fig. 12. One sees that, despite a rather strong difference in the radial structure, the zonal flows evolution is similar for small $k=2$ and "large" $k=5$ "wave numbers". This is in accordance both with our expectations and with results of Ref. [17]. The situation is quite different if the Mach number becomes very small. In this case, the evolution is shown in Fig. 13 and the radial profiles of $\phi$ are plotted in Fig. 14. One sees an obvious sensitivity of the zonal flow to the characteristic radial scale of the perturbation at small/vanishing ambient radial electric field. This is consistent with findings of Refs. $[8,13]$.

In all simulations shown above we have assumed the ambient electric field to be constant (flat). What will happen, however, if we relax this limitation? The answer is shown in Fig. 15. Here, the zonal flow evolution at the Mach number $u_{E} / v_{\text {th } i}=0.018$ is compared for the ambient radial electric field profiles shown in Fig. 16 (one profile is flat, another is proportional to the toroidal flux). One sees that the non-oscillatory part remains almost unchanged, in contrast to the zonal flow oscillations, which are strongly damped. The nature of this damping is indicated in Eq. (18). Since $\phi \sim \cos \left(\omega_{Z F} t\right)$ with $\omega_{Z F} \approx \Omega_{E}(s)$, it is clear that a phase mixing can occur in real space if the ambient electric field has a finite shear. The associated damping mechanism is reminiscent of the well-known continuum damping of the shear Alfvén waves [25-27]. An appropriate analytical treatment of this problem must start from the initial-value formulation and involve solution of an inhomogeneous boundary-value problem. We do not consider it here.

Finally, let us consider which effect the magnetic geometry can have on the zonal flow evolution. All the results above were obtained in the magnetic field of W7-X, which is drift-optimized. Now, we perform our simulations in a more "classical" heliotron magnetic geometry (similar to the LHD configuration with the major radius $R_{0}=3.75 \mathrm{~m}$ ). The results are shown in Fig. 17. Here, the zonal flow evolution at different radial positions is shown for the Mach number $u_{E} / v_{\text {th } i}=0.025$ (flat profile). Again we can see GAMs (which are quite strong in LHD). The zonal flow oscillation mode, however, can hardly be seen (although 
it is present and has properties similar to what have been observed in the standard W7-X geometry $[13,14])$. A possible explanation for this difference is due to the bounce-averaged poloidal magnetic drift frequency, which is larger in a conventional stellarator geometry compared to the drift-optimized configurations [3]. As a result, the algebraic damping of the zonal flow mode dominates its evoulution. The residual level is also clearly smaller in conventional stellarator/heliotron geometry than it is in optimized geometries [this can be seen from Eq. (18) noting that $\left.d_{r} \sim V_{r} / \Omega_{E}\right]$.

\section{DISCUSSION AND CONCLUSIONS}

In this paper we have studied the effect of the radial electric field on the zonal flow (linear collisionless dynamics) in stellarator geometry. A radial neoclassical electric field is always present in stellarators because the neoclassical transport is not automatically ambipolar in non-axisymmetric magnetic field [28]. Thus, studying the effect of this field on the residual zonal flow should have a practical relevance. Note that there are, in principle, certain means to affect the neoclassical electric field (and, consequently, the zonal flow) in experiments (for example, applying Electron Cyclotron Heating in the plasma center etc).

Two different configurations have been considered representing distinct types of the stellarator design: a heliotron configuration and the drift-optimized geometry of W7-X. Similarly to Refs. $[14,17,18]$, we have found that the ambient radial electric field strongly affects the residual level, which increases with the electric field. This phenomenon is caused by the reduction of the poloidal-drift orbit width with increasing radial electric field. Clearly, the effect depends on the magnetic drift velocity and, hence, can strongly be affected by the magnetic geometry, what indeed has been observed in our simulations: the residual in the drift-optimized W7-X was larger than in LHD at similar ambient electric fields. Another difference between zonal flows in these devices is the transient dynamics (the zonal flow oscillations), which is clearly observed in W7-X (and may be important since the associated time scales can be comparable to the nonlinear correlation time). In LHD, this transient dynamics is strongly damped, which may also be caused by the larger magnetic drift velocity there.

In our simulations, we have seen that the profile of the ambient electric field (its shear) is important for the zonal-flow evolution, too. Finite shear causes continuum damping of 
the zonal flow oscillations (but does not strongly affect the residual). Global simulations are probably needed to observe such an effect. This dependence on the electric field profile is interesting since a variety of profiles are possible for neoclassical electric fields in stellarators (depending on plasma properties, e. g. the pressure profile, collisional regimes etc). Note that also direction of the radial electric field will influence the zonal flow response. Physically, it determines the direction of the poloidal $\mathbf{E} \times \mathbf{B}$ drift which can either be in the direction of the magnetic drift or in the opposite one. This effect has been discussed in Ref. [17]. It has also been observed numerically in our simulations (not shown here).

Concluding, the linear zonal flow dynamics in non-axisymmetric geometry is considerably more complicated than it is in tokamaks. The details of this dynamics depend strongly on the particular stellarator type considered. In addition to the asymptotic residual level, transient effects such as zonal-flow oscillations may be of practical importance unless they are strongly damped as is the case in LHD. The neoclassical electric field is an important control parameter which can substantially affect the zonal flow physics in stellarators. In addition, collisional and, of course, nonlinear phenomena are of importance on the time scales considered (e. g. transient times). The associated physics remains to be studied.

In the outlook, we believe that the quantitative numerical study of the zonal flows in stellarators must be supported by numerical benchmarks between different gyrokinetic codes capable to simulate them in the global or full-surface non-axisymmetric geometry. Such a benchmark has already been successfully undertaken in Ref. [15] in the stellarator flux-tube geometry (GKV results [8] have been compared with GENE). A global-geometry (or full-surface) benchmark has been beyond the scope of the present paper but it is an important piece of work for the next step. We believe that both the understanding of the zonal flow physics and the associated numerical framework would benefit from this effort.

\section{ACKNOWLEDGEMENTS}

We acknowledge support of P. Helander for this work. The simulations were performed using the DEISA supercomputing grid infrastructure, the HPC-FF (Forschungszentrum Jülich) and Blue Gene/P (Rechenzentrum Garching) supercomputers. Support of the High Level 
Support Team (Garching) on the code optimization is appreciated.

[1] P. H. Diamond, S.-I. Itoh, K. Itoh, and T. S. Hahm, Plasma Phys. Controlled Fusion 47, R35 (2005).

[2] J. Nuehrenberg and R. Zille, Phys. Lett. A 129, 113 (1988).

[3] H. E. Mynick, Phys. Plasmas 13, 058102 (2006).

[4] H. E. Mynick, N. Pomphrey, and P. Xanthopoulos, Phys. Rev. Lett. 105, 095004 (2010).

[5] M. Rosenbluth and F. Hinton, Phys. Rev. Lett. 80, 724 (1998).

[6] Y. Xiao and P. Catto, Phys. Plasmas 13, 082307 (2006).

[7] Y. Xiao, P. Catto, and W. Dorland, Phys. Plasmas 14, 055910 (2007).

[8] H. Sugama and T.-H. Watanabe, Phys. Plasmas 13, 012501 (2006).

[9] O. Motojima, N. Ohyabu, A. Komori, O. Kaneko, S. Masuzaki, A. Ejiri, M. Emoto, H. Funaba, M. Goto, K. Ida, H. Idei, S. Inagaki, N. Inoue, S. Kado, S. Kubo, R. Kumazawa, T. Minami, J. Miyazawa, T. Morisaki, S. Morita, S. Murakami, S. Muto, T. Mutoh, Y. Nagayama, Y. Nakamura, H. Nakanishi, K. Narihara, K. Nishimura, N. Noda, T. Kobuchi, S. Ohdachi, Y. Oka, M. Osakabe, T. Ozaki, B. J. Peterson, A. Sagara, S. Sakakibara, R. Sakamoto, H. Sasao, M. Sasao, K. Sato, M. Sato, T. Seki, T. Shimozuma, M. Shoji, H. Suzuki, Y. Takeiri, K. Tanaka, K. Toi, T. Tokuzawa, K. Tsumori, K. Tsuzuki, I. Yamada, S. Yamaguchi, M. Yokoyama, K. Y. Watanabe, T. Watari, Y. Hamada, K. Matsuoka, K. Murai, K. Ohkubo, I. Ohtake, M. Okamoto, S. Satoh, T. Satow, S. Sudo, S. Tanahashi, K. Yamazaki, M. Fujiwara and A. Iiyoshi, Nucl. Fusion 43, 1674 (2003).

[10] H. Sugama and T.-H. Watanabe, Phys. Rev. Lett. 94, 115001 (2005).

[11] S. Ferrando-Margalet, H. Sugama, and T.-H. Watanabe, Phys. Plasmas 14, 122505 (2007).

[12] A. Mishchenko, P. Helander, and A. Könies, Phys. Plasmas 15, 072309 (2008).

[13] P. Helander, A. Mishchenko, R. Kleiber, and P. Xanthopoulos, Plasma Phys. Controlled Fusion 53, 054006 (2011).

[14] R. Kleiber, R. Hatzky, and A. Mischchenko, Contrib. Plasma Phys. 50, 766 (2010).

[15] P. Xanthopoulos, A. Mischchenko, P. Helander, H. Sugama, and T. H. Watanabe, Phys. Rev. Lett. 107, 245002 (2011).

[16] G. Grieger, C. D. Beidler, H. Maassberg, E. Harmeyer, F. Herrnegger, J. Junker, J. Kisslinger, 
W. Lotz, P. Merkel, J. Nührenberg, F. Rau, J. Sapper, A. Schlüter, F. Sardei, and H. Wobig, in Proc. of the 13th International Conference on Plasma Physics and Controlled Nuclear Fusion Research (International Atomic Energy Agency, Vienna, 1991), Vol. 3, p. 525.

[17] H. Sugama and T.-H. Watanabe, Phys. Plasmas 16, 056101 (2009).

[18] H. E. Mynick and A. H. Boozer, Phys. Plasmas 14, 072507 (2007).

[19] H. Sugama and T.-H. Watanabe, Contrib. Plasma Phys. 50, 571 (2010).

[20] T. H. Watanabe, H. Sugama, and M. Nunami, Nucl. Fusion 51, 123003 (2011).

[21] V. Kornilov, R. Kleiber, R. Hatzky, L. Villard, and G. Jost, Phys. Plasmas 11, 3196 (2004).

[22] A. H. Boozer, Phys. Fluids 25, 520 (1982).

[23] A. H. Boozer and H. J. Gardner, Phys. Fluids B 2, 2408 (1990).

[24] M. Wakatani, Stellarator and Heliotron Devices (Oxford University Press, Oxford, 1998).

[25] F. Zonca and L. Chen, Phys. Rev. Lett. 68, 592 (1992).

[26] H. L. Berk, J. W. V. Dam, Z. Guo, and D. M. Lindberg, Phys. Fluids B 4, 1806 (1992).

[27] M. N. Rosenbluth, H. L. Berk, J. W. V. Dam, and D. M. Lindberg, Phys. Fluids B 4, 2189 (1992).

[28] P. Helander and A. Simakov, Phys. Rev. Lett. 101, 145003 (2008). 


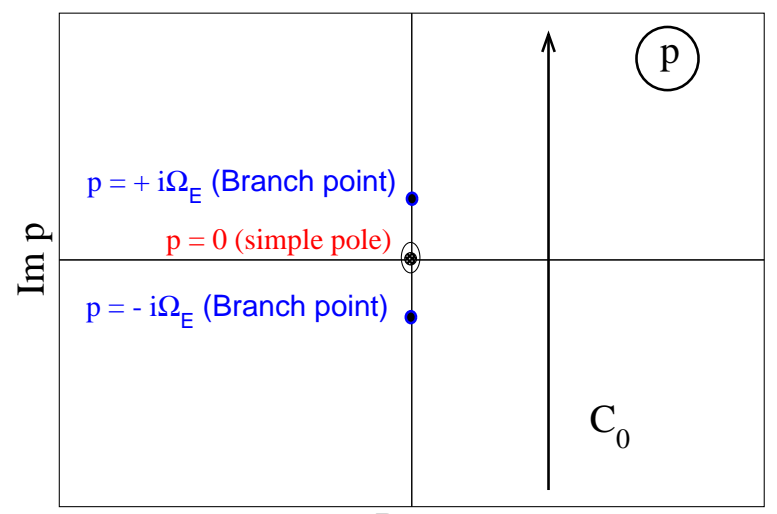

$\operatorname{Re} \mathrm{p}$

FIG. 1: (Color online): Initial integration contour in the Laplace transform. 


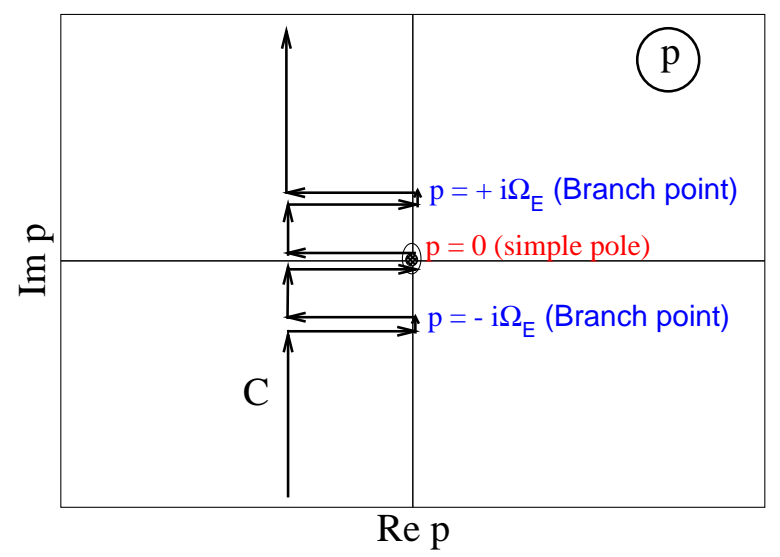

FIG. 2: (Color online): Deformed integration contour in the Laplace transform. 




FIG. 3: (Color online): Lower cut in the inverse Laplace transform integration contour. 


\section{Upper branch (Lower cut)}



FIG. 4: (Color online): Integration contour in the velocity space corresponding to the analytic continuation on the upper branch of the lower cut in the inverse Laplace transform. 
Lower branch (Lower cut)

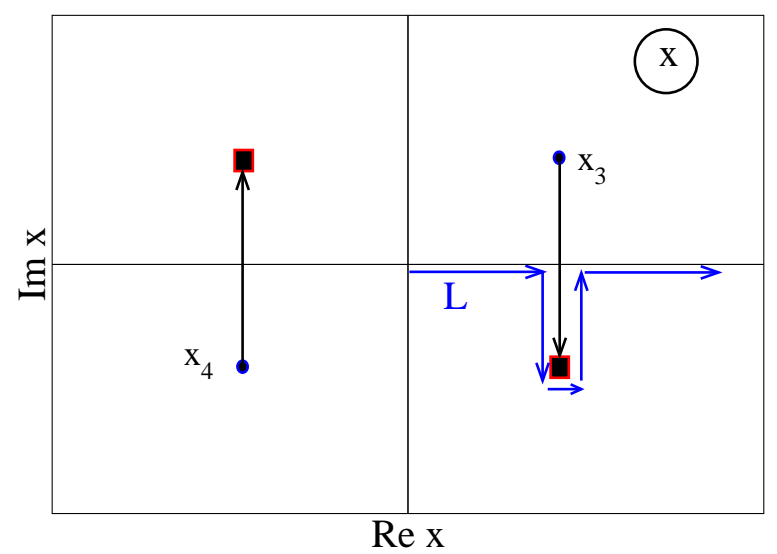

FIG. 5: (Color online): Integration contour in the velocity space corresponding to the analytic continuation on the lower branch of the lower cut in the inverse Laplace transform. 


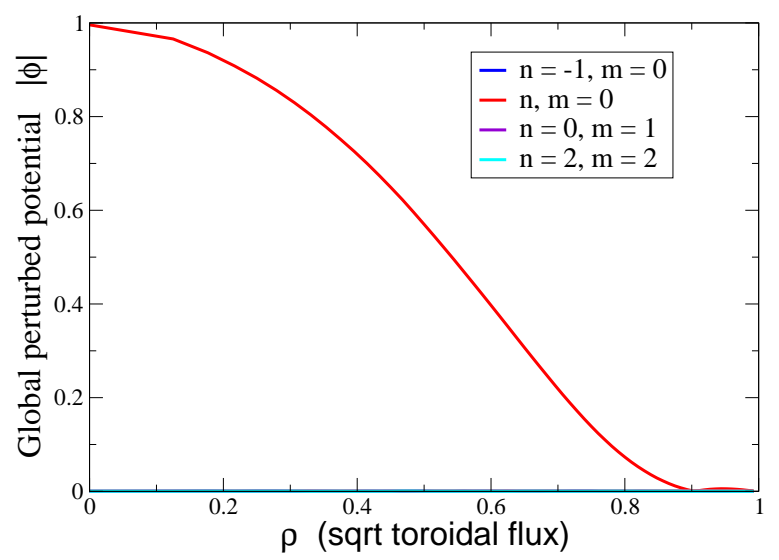

FIG. 6: (Color online): Global radial profile of the perturbed electrostatic potential. One sees that the zonal component is dominant. 


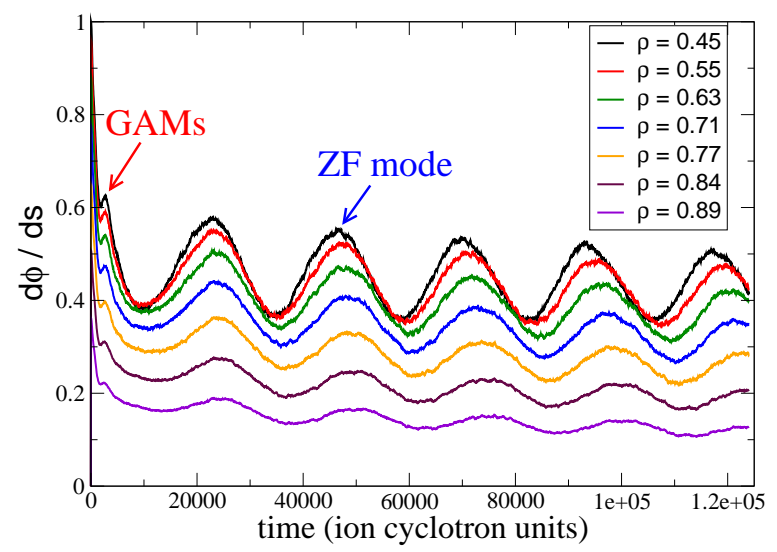

FIG. 7: (Color online): Time evolution of $\partial \phi / \partial s$ (zonal component) at different flux surfaces. 
$\mathrm{ZF}$ at position $\mathrm{s}=0.55$

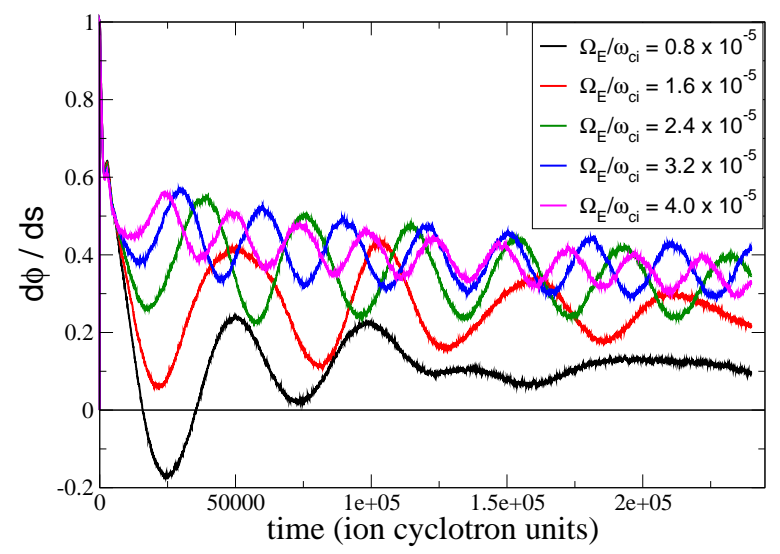

FIG. 8: (Color online): Zonal flow evolution pattern at the same radial position as a function of the ambient electric field. 
Residual potential

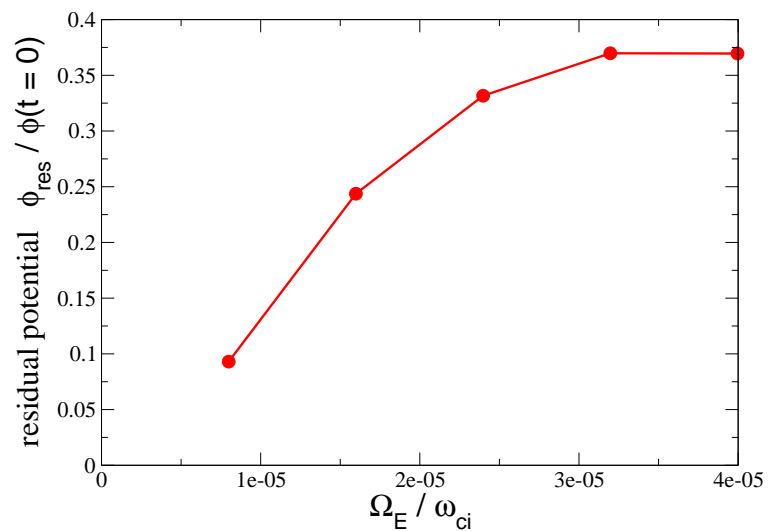

FIG. 9: (Color online): Zonal flow residual as a function of the ambient electric field. 


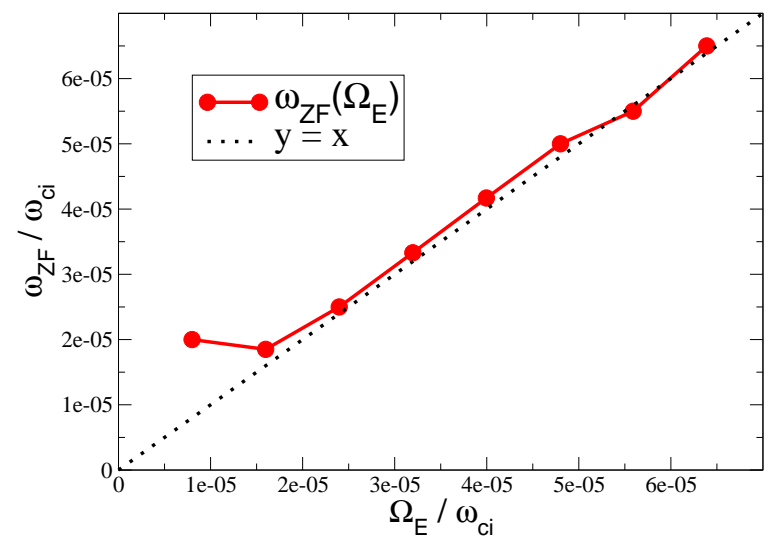

FIG. 10: (Color online): Zonal flow frequency as a function of the ambient electric field. 


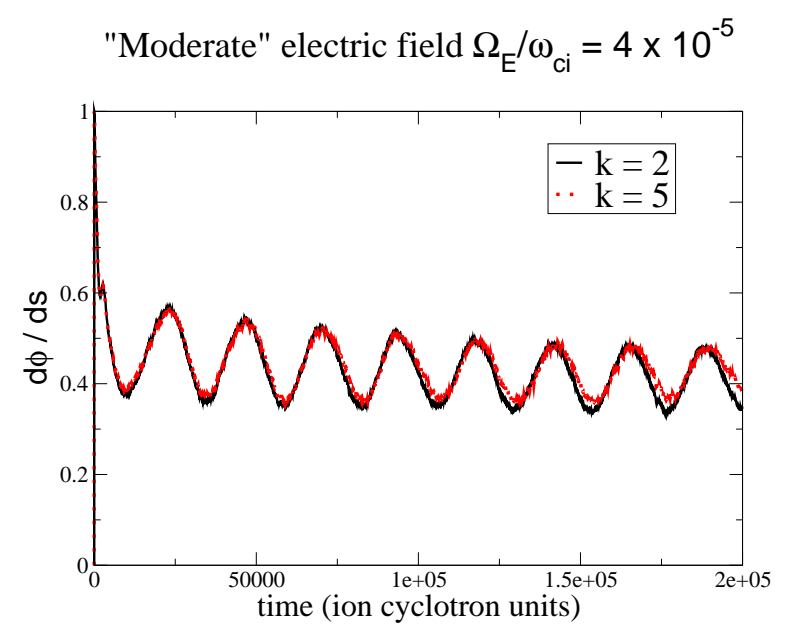

FIG. 11: (Color online): Zonal flow evolution at "moderate" Mach number does not depend on the "radial wave number". 
"Moderate" electric field $\Omega_{\mathrm{E}} / \omega_{\mathrm{ci}}=4 \times 10^{-5}$

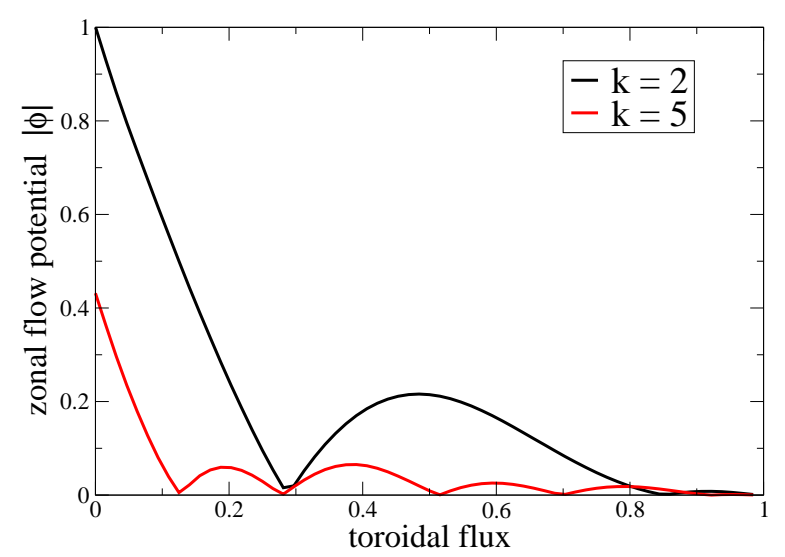

FIG. 12: (Color online): Global radial profiles of the perturbed electrostatic potential for different "radial wave numbers" at moderate $\Omega_{E}$. 




FIG. 13: (Color online): Zonal flow evolution at small Mach number is sensitive with respect to the "radial wave number". 
Small electric field $\Omega_{\mathrm{E}} / \omega_{\mathrm{Ci}}=0.8 \times 10^{-5}$

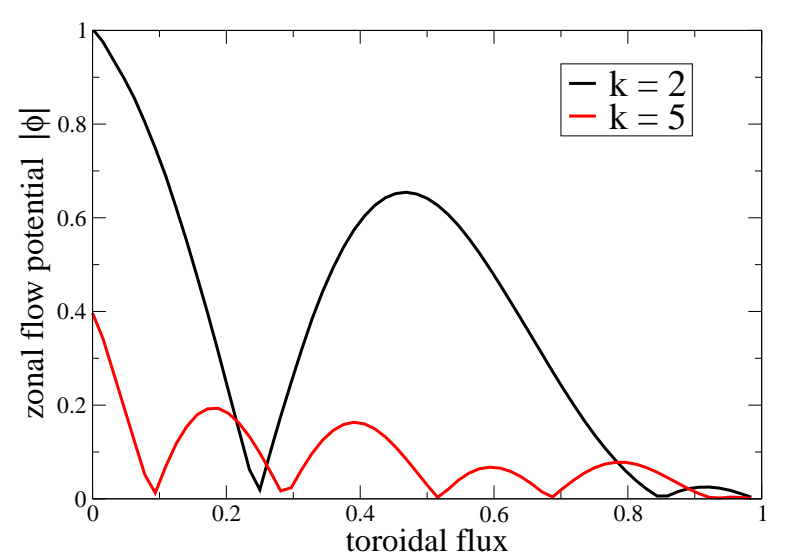

FIG. 14: (Color online): Global radial profiles of the perturbed electrostatic potential for different "radial wave numbers" at small $\Omega_{E}$. 


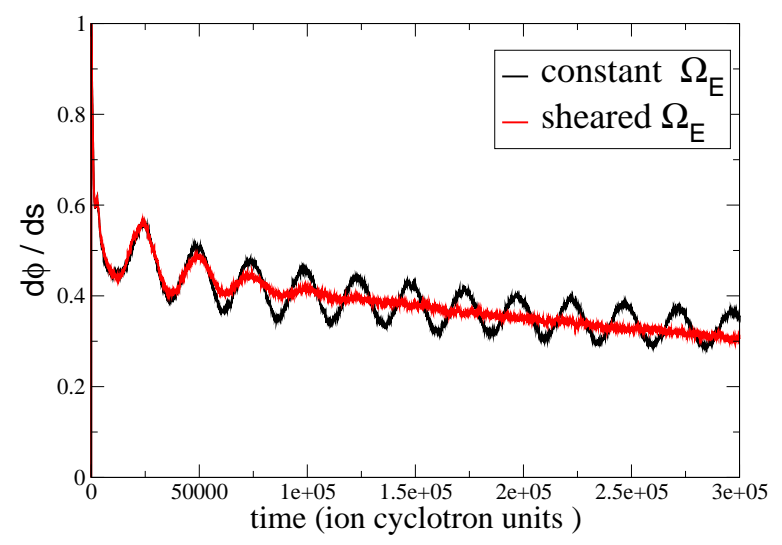

FIG. 15: (Color online): Zonal flow evolution; flat ambient electric field profile case compared to evolution employing profile with finite shear (see Fig. 16). 


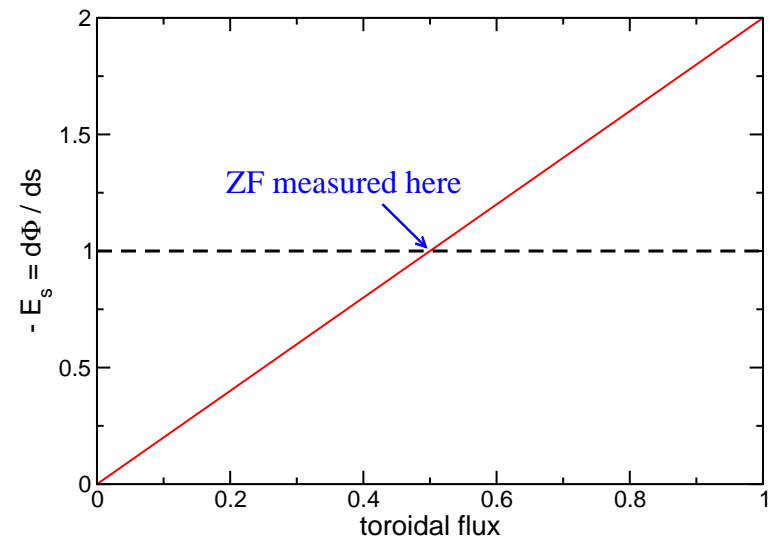

FIG. 16: (Color online): Ambient radial electric field profiles used for simulations shown in Fig. 15. 


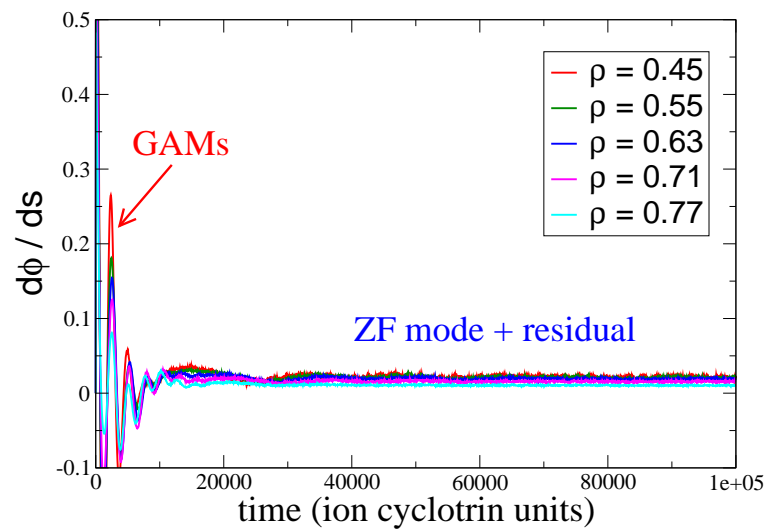

FIG. 17: (Color online): Zonal flow evolution in LHD at the Mach number $u_{E} / v_{\text {th } i}=0.025$. 